\title{
An Automatic Electropolishing System for Needle-Shaped Specimens
}

\author{
S.L.P. Kostrna*, J.W. Peterman**, T.J. Prosa*, S.A. Wiener* D.J. Larson*, and T.F. \\ Kelly* \\ *Imago Scientific Instruments Corporation, 6300 Enterprise Lane, Madison, WI 53719 \\ USA \\ **Simplex Scientific, 1910 Mayflower Drive, Middleton, WI 53562 USA
}

\begin{abstract}
Although the electropolishing of metals is based in science, there has always been a hint of art involved [1]. The development of an automated polisher has been pursued in order to make specimen preparation for atom probe and other metrologies more accessible and reproducible. The goals have been to develop a computer controlled process to repeat successful polishing routines and to develop an automated, all-in-one tool that performs both rough and fine polishing operations, saving users' time.
\end{abstract}

The basics of metal electropolishing are well known: a metal specimen is placed in an electrolytic solution while another piece of relatively-inert metal - usually platinum - is placed in proximity to the specimen and a voltage is applied to the circuit [2]. Material is dissolved from the specimen, functioning as an anode, and pulled toward inert metal, which serves as the cathode, to create a desired shape. The basic electropolishing process is straight-forward and a variety of self-engineered setups exist in laboratories throughout the world.

Existing instrumentation is not able to produce a finely-polished, ready-to-run atom probe specimen automatically, so a multiple-step process is undertaken, starting with rough polishing. To begin rough polishing, a several-millimeter-long specimen is polished until a necking region is formed (Fig. 1). This necking region needs to be long enough to be seen by the operator. Rough polishing is stopped immediately when the necking region breaks off, otherwise the speed of the rough polishing process very quickly dulls the apex and forces a repeat of this step.

To automate this procedure, a current-monitoring device replaces visual anticipation and confirmation of the breaking of the necking region. Micro-polishing the specimen to its final shape begins after rough polishing.

A specimen that is sharp enough for atom probe analysis $-<200 \mathrm{~nm}$ diameter at apex will be too sharp to resolve with a light microscope. However, a typical procedure is to polish until the apex can no longer be focused by such an instrument, indicating that the tip is sharper than the resolution of the available optics. This highly hands-on process, coupled with limited ability to judge adequate final tip dimension, becomes the "Art of Electropolishing" which intimidates the uninitiated and haunts the experienced.

The Simplex Scientific ElectroPointer ${ }^{\mathrm{TM}}$ offers an answer to the vagaries of electropolishing and includes automated control of polishing parameters and a hands-off approach that minimizes the need for subjective decision-making. The computer interface allows input of basic parameters to achieve a repeatable tip shape with minimal previous experience. For basic operations, the user can set voltage level, polishing pass width and speed. More advanced operations allow the user to define the number of passes 
completed before the supply of electrolyte is refreshed (Fig. 2). During polishing, current and time are tracked for informational purposes. A particularly useful feature for untested specimens is a current vs. voltage measurement allows the identification of the optimal polishing range for a given specimen type and electrolyte. This graph follows the etch/polish/pit curve commonly cited on this subject [2].

Several options are available for determining when a specimen is complete: total number of passes, integrated charge, minimum current and manual stopping. An automated endpoint detection method is currently in development to sense when the neck has dropped and a specimen tip of appropriate dimension has formed. To further aid in controlling the diameter of a specimen, a manual pulse mode may be required. Pulse duration from one to one hundred microseconds offer nanometer-scale control in final polishing. This feature is especially useful for dulling too-sharp specimens to create a large-field of view atom probe specimen and for positioning features of interest near the apex for subsequent analysis (Fig. 3).

\section{References:}

[1] A.J. Melmed, J.V.S.T. B9 (1991) 601.

[2] D.B. Williams, C.B. Carter, Transmission Electron Microscopy, Plenum Press, New York, 1996.

[3] This work is supported by the National Science Foundation, SBIR Phase II Grant No. 0216620 .

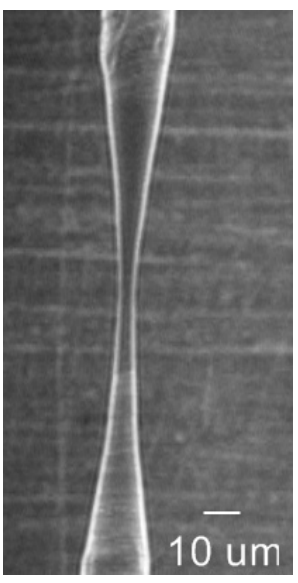

Fig. 1

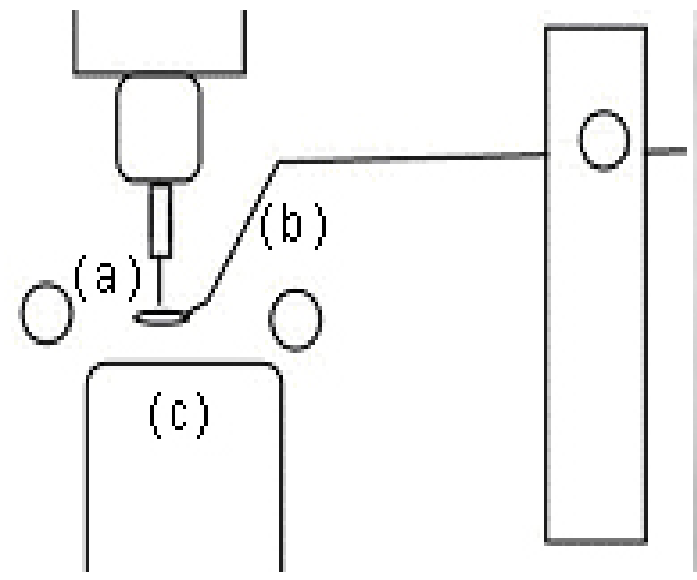

Fig. 2

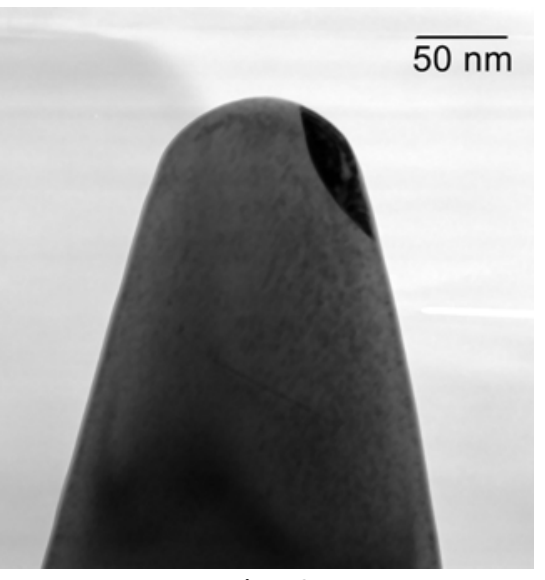

Fig. 3

Fig. 1. A specimen in the process of being "necked." Rough polishing creates a narrow region from which a tip ready for micro-polishing will be formed.

Fig. 2. The ElectroPointer comprising a stationary specimen (a) with a moveable counterelectrode loop (b) and electrolyte (c). The loop travels between specimen and electrolyte during electropolishing.

Fig. 3. Transmission Electron Micrograph of an atom probe specimen. The specimen has been polished to bring the grain boundary at right into the area of analysis at the tip. 